\title{
Computing the Effective Hamiltonian using a Variational Approach
}

\author{
Diogo A. Gomes and Adam M. Oberman
}

\begin{abstract}
A numerical method for homogenization of Hamilton-Jacobi equations is presented and implemented as an $L^{\infty}$ calculus of variations problem. Solutions are found by solving a nonlinear convex optimization problem. The numerical method is shown to be convergent and error estimates are provided. Several examples are worked in detail, including the cases of non-strictly convex Hamiltonians and Hamiltonians for which the cell problem has no solution.
\end{abstract}

\section{INTRODUCTION}

Given the Hamiltonian $H(p, x)$ which is smooth, convex in $p$, and periodic in the second variable $x$, we consider, for a given $P \in \mathbb{R}^{n}$, periodic solutions of the Hamilton-Jacobi equation

$$
H\left(P+D_{x} u, x\right)=\bar{H}(P) .
$$

For each fixed $P$ the problem (HB) can be regarded as a nonlinear eigenvalue problem for the function function $u(x)$ and the number $\bar{H}(P)$, the effective Hamiltonian.

In this paper, we reduce the problem of finding the (approximate) effective Hamiltonian to a finite dimensional convex optimization problem, which may solved numerically using standard methods.

Numerical computations of effective Hamiltonians have been done by [EMS95], [KBM01], with applications to front propagation and combustion, and in [Qia01], both of them using partial differential equations methods.

In this work we circumvent the difficulties of solving (HB) by computing $\bar{H}(P)$ without finding the solution $u$. Our methods are based on the representation formula

$$
\bar{H}(P)=\inf _{\phi \in C_{p e r}^{1}} \sup _{x} H\left(P+D_{x} \phi, x\right)
$$

due, for strictly convex Hamiltonians, to [CIPP98].

In this paper we always assume that $H$ to be convex but not necessarily strictly convex. This assumption has implications for the existence and smoothness of solutions of (HB). If strict convexity fails, solutions may (see $\S I V-B$ ) or may not (see $\S I V-C$ ) exist, and the degree of smoothness will depend on the Hamiltonian in question.

Computing the effective Hamiltonian is relevant in several problems, as we describe briefly next.

In homogenization problems [LPV88], [Con95], if $w^{\epsilon}$ solves

$$
-w_{t}^{\epsilon}+H\left(D_{x} w^{\epsilon}, \frac{x}{\epsilon}\right)=0,
$$

then as $\epsilon$ goes to 0 , the solution $w^{\epsilon}$ converges to $w^{0}$ which is a solution of the limiting problem

$$
-w_{t}^{0}+\bar{H}\left(D_{x} w^{0}\right)=0 .
$$

DG was partially supported by FCT/POCTI/FEDER and
In the study of long time limits of viscosity solutions of Hamilton-Jacobi equations

$$
-w_{t}+H\left(P+D_{x} w, x\right)=0,
$$

the difference $w(x, t)-\bar{H}(P) t$ converges as $t \rightarrow-\infty$ to a stationary solution of (HB) [Fat98b], [BS00]. See also [AI01], [CDI01].

In classical mechanics smooth solutions $u$ of $(\mathrm{HB})$ yield a canonical change of coordinates $X(p, x)$ and $P(p, x):(p=$ $P+D_{x} u, X=x+D_{P} u$ ), which simplifies the Hamiltonian dynamics

$$
\dot{x}=-D_{p} H(p, x) \quad \dot{p}=D_{x} H(p, x)
$$

into the trivial dynamics

$$
\dot{P}=0 \quad \dot{X}=-D_{P} \bar{H}(P) .
$$

In Aubry-Mather theory [Mat89], [Mat91], one looks for probability measures $\mu$ on $\mathbb{T}^{n} \times \mathbb{R}^{n}$ that minimize

$$
\int L(x, v)+P \cdot v d \mu
$$

where $L(x, v)$ is the Legendre transform of $H(p, x)$, and satisfy a holonomy condition:

$$
\int v D_{x} \phi d \mu=0, \text { for all } \phi(x) \in C^{1}\left(\mathbb{T}^{n}\right) .
$$

The supports of these measures are called the Aubry-Mather sets, [E99], [Fat97a], [Fat97b], [Fat98a], [Fat98b], [CIPP98], [EG01a], [EG01b], [Gom01b]. Viscosity solutions of (HB) encode important properties of the Aubry-Mather sets. In particular,

$$
\int L(x, v)+P \cdot v d \mu=-\bar{H}(P),
$$

the support of the Mather measure is a subset of the graph

$$
\left(x,-D_{p} H\left(P+D_{x} u, x\right)\right),
$$

for any viscosity solution of (HB), and if $(x, p)$ belongs to any Mather, and $(x(t), p(t))$ is its orbit under (2) then

$$
\frac{x(T)}{T} \rightarrow-D_{P} \bar{H}(P),
$$

for some $P$, if $\bar{H}$ is differentiable.

Equation (HB) and related stationary first and second order Hamilton-Jacobi equations are also important to the ergodic control problem [Ari98], [Ari97]. Effective Hamiltonians also arise in the study of propagation of flame fronts in combustion: in this case, solving a homogenization problem gives the effective or averaged front speed [EMS95], [KBM01]. 


\section{Convergence Results}

We start this section by reviewing two results concerning the function $\bar{H}(P)$ :

Proposition 1 (Lions, Papanicolao, Varadhan): There is at most one value $\bar{H}$ for which (HB) has a periodic viscosity solution.

Proposition 2 (Contreras, Iturriaga, Paternain, Paternain): Suppose $H$ is periodic in $x$ and convex in $p$. Assume that there exists a viscosity solution $u$ of (HB). Then

$$
\bar{H}=\inf _{\psi \in C^{1}\left(\mathbb{T}^{n}\right)} \sup _{x \in \mathbb{T}^{n}} H\left(D_{x} \psi, x\right),
$$

in which the infimum is taken over the space $C^{1}\left(\mathbb{T}^{n}\right)$ of periodic functions.

We should note that the original proof required strict convexity, but a simple viscosity solution argument overcomes this problem.

The next issue is the approximation of the problem (1). To this effect, consider a triangulation of $\mathbb{T}^{n}$ with cells of diameter smaller than $h$. Let $C\left(T_{h}\right)$ be the collection of piecewise linear finite elements which interpolate given nodal values.

Proposition 3: Suppose $H(p, x)$ is convex in $p$. Then $\inf _{\psi \in C^{1}\left(\mathbb{T}^{n}\right)} \sup _{x} H\left(D_{x} \psi, x\right)=\lim _{h \rightarrow 0} \inf _{\phi \in C\left(T_{h}\right)} \operatorname{esssup} H\left(D_{x} \phi, x\right)$. Proof: Fix $\epsilon>0$. Let $\psi$ be a $C^{1}$ function for which

$$
\sup _{x \in \mathbb{T}^{n}} H\left(D_{x} \psi, x\right) \leq \inf _{\psi \in C^{1}\left(\mathbb{T}^{n}\right)} \sup _{x \in \mathbb{T}^{n}} H\left(D_{x} \psi, x\right)+\epsilon .
$$

Because $\psi$ is $C^{1}, D_{x} \psi$ is uniformly continuous. Thus, for $h$ sufficiently small, there is $\phi \in C\left(T_{h}\right)$ such that $\operatorname{esssup}\left|D_{x} \phi-D_{x} \psi\right| \leq \epsilon$. This implies $x \in \mathbb{T}^{n}$

$$
\underset{x \in \mathbb{T}^{n}}{\operatorname{esssup}} H\left(D_{x} \phi, x\right) \leq \sup _{x \in \mathbb{T}^{n}} H\left(D_{x} \psi, x\right)+O(\epsilon),
$$

by Lipschitz continuity of $H$ in $p$. Thus, taking first $\lim _{h \rightarrow 0} \inf _{\phi \in C\left(T_{h}\right)}$, then $\inf _{\psi \in C^{1}\left(\mathbb{T}^{n}\right)}$, and finally $\epsilon \rightarrow 0$, we obtain the first inequality.

To prove the converse inequality observe that if $\phi \in$ $C\left(T_{h}\right), \eta_{\epsilon}$ is a smooth mollifier, and $\psi=\eta_{\epsilon} * \phi$, then convexity yields

$$
H\left(D_{x} \psi(x), x\right) \leq \int H\left(D_{x} \phi(y), y\right) \eta_{\epsilon}(x-y) d y+O(\epsilon),
$$

every $x$, and so the result follows from

$$
H\left(D_{x} \psi(x), x\right) \leq \underset{x \in \mathbb{T}^{n}}{\operatorname{esssup}} H\left(D_{x} \phi(x), x\right)+O(\epsilon),
$$

taking first $\inf _{\psi \in C^{1}}$, then $\lim _{h \rightarrow 0} \inf _{\phi \in C\left(T_{h}\right)}$, and finally $\epsilon \rightarrow 0$.

First observe that

$$
\mathcal{H}(\phi)=\sup _{x \in \mathbb{T}^{n}} H\left(D_{x} \phi, x\right),
$$

is a convex, but not strictly convex, functional. Therefore local minima are global minima.

Proposition 4: The approximate Hamiltonian

$$
\bar{H}_{h}(P)=\inf _{\phi \in C\left(T_{h}\right)} \underset{x \in \mathbb{T}^{n}}{\operatorname{esssup}} H\left(P+D_{x} \phi, x\right)
$$

is convex in $P$.

Proof: Let $P_{1}, P_{2} \in \mathbb{R}^{n}$ and let $\phi_{1}, \phi_{2} \in C\left(T_{h}\right)$ be the corresponding minimizers. Let $0 \leq \lambda \leq 1$, and set $P=$ $\lambda P_{1}+(1-\lambda) P_{2}$, and $\phi=\lambda \phi_{1}+(1-\lambda) \phi_{2}$. Then, for any $x$ we have

$$
\begin{aligned}
& H\left(P+D_{x} \phi, x\right) \\
& \quad \leq \lambda H\left(P_{1}+D_{x} \phi_{1}, x\right)+(1-\lambda) H\left(P_{2}+D_{x} \phi_{2}, x\right),
\end{aligned}
$$

and so

$$
\begin{aligned}
\bar{H}_{h}(P) & =\inf _{\phi \in C\left(T_{h}\right)} \underset{x \in \mathbb{T}^{n}}{\operatorname{esssup}} H\left(P+D_{x} \phi, x\right) \\
& \leq \lambda \bar{H}_{h}\left(P_{1}\right)+(1-\lambda) \bar{H}_{h}\left(P_{2}\right) .
\end{aligned}
$$

Theorem 1: For any convex Hamiltonian $H(p, x)$ for which (HB) has a viscosity solution

$$
\bar{H} \leq \inf _{\phi \in C\left(T_{h}\right)} \operatorname{esssup} H\left(D_{x} \phi, x\right) .
$$

If there exists a globally $C^{2}$ solution of (HB) then

$$
\inf _{\phi \in C\left(T_{h}\right)} \operatorname{esssup} H\left(D_{x} \phi, x\right)=\bar{H}+O(h) .
$$

If (HB) has a Lipschitz solution (for instance if $H(p, x)$ is strictly convex in $p$ ) we have

$$
\inf _{\phi \in C\left(T_{h}\right)} \underset{x}{\operatorname{esssup}} H\left(D_{x} \phi, x\right)=\bar{H}+O\left(h^{1 / 2}\right) .
$$

If $H$ is convex but not strictly convex and (HB) has a viscosity solution then

$$
\inf _{\phi \in C\left(T_{h}\right)} \operatorname{esssup}_{x} H\left(D_{x} \phi, x\right)=\bar{H}+o(1) .
$$

Proof: Observe that

$\bar{H}=\inf _{\psi \in C^{1}\left(\mathbb{T}^{n}\right)} \sup _{x} H\left(D_{x} \psi, x\right) \leq \inf _{\phi \in C\left(T_{h}\right)} \operatorname{esssup} H\left(D_{x} \phi, x\right)$,

because by convexity we can associate to each $\phi \in C\left(T_{h}\right)$ a function $\psi=\phi * \eta_{\epsilon} \in C^{1}\left(\mathbb{T}^{n}\right)$ such that $\sup _{x} H\left(D_{x} \psi, x\right) \leq$ $\operatorname{esssup} H\left(D_{x} \phi, x\right)+O(\epsilon)$, for arbitrary $\epsilon>0$.

To prove the second assertion suppose $u$ is a $C^{2}$ viscosity solution of (HB). Fix $h$ and construct a function $\phi_{u} \in C\left(T_{h}\right)$ by interpolating linearly the values of $u$ at the nodal points. In each triangle $T^{i}$, the oscillation of the derivative of $u$ is $O(h)$, since $u$ is $C^{2}$. Thus, we obtain

$$
D_{x} \phi_{u}(x)=D_{x} u(x)+O(h),
$$

for any $x$. Since $H\left(D_{x} u, x\right)=\bar{H}$, at every point $x \in T^{i}$ we have $H\left(D_{x} \phi_{u}, x\right) \leq \bar{H}+O(h)$, which implies

$$
\inf _{\phi \in C\left(T_{h}\right)} \operatorname{esssup}_{x \in \mathbb{T}^{n}} H\left(D_{x} \phi, x\right) \leq \bar{H}+O(h) .
$$

If $u$ is a Lipschitz viscosity solution, let $\tilde{u}=\eta_{h^{1 / 2}} * u$. Observe that $\left|D_{x x}^{2} \tilde{u}\right| \leq \frac{C}{h^{1 / 2}}$, and

$$
H\left(D_{x} \tilde{u}, x\right) \leq \bar{H}+O\left(h^{1 / 2}\right) .
$$

Construct a function $\phi_{u} \in C\left(T_{h}\right)$ by interpolating linearly the values of $\tilde{u}$ at the nodal points. In each triangle $T^{i}$, the oscillation of the derivative of $\tilde{u}$ is $O\left(h^{1 / 2}\right)$. 


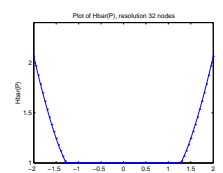

Fig. 1: $\bar{H}(P)$ for the one-dimensional pendulum.

Thus $D_{x} \phi_{u}(x)=D_{x} \tilde{u}(x)+O\left(h^{1 / 2}\right)$, for any $x$. Since $H\left(D_{x} \tilde{u}, x\right) \leq \bar{H}+O\left(h^{1 / 2}\right)$, for every point $x \in T^{i}$ we have $H\left(D_{x} \phi_{u}, x\right) \leq \bar{H}+O\left(h^{1 / 2}\right)$. This implies

$$
\inf _{\phi \in C\left(T_{h}\right)} \operatorname{esssup}_{x \in \mathbb{T}^{n}} H\left(D_{x} \phi, x\right) \leq \bar{H}+O\left(h^{1 / 2}\right) .
$$

The last case, for not strictly convex Hamiltonians, the sup convolution yields a function $u_{h^{1 / 3}}$ that satisfies

$$
H\left(D_{x} u_{h^{1 / 3}}, x\right) \leq \bar{H}+o(1)
$$

almost everywhere and has Lipschitz constant bounded by $\frac{C}{h^{1 / 3}}$. Define $\tilde{u}=\eta_{h^{1 / 3}} * u_{h^{1 / 3}}$ which satisfies $H\left(D_{x} \tilde{u}, x\right) \leq$ $\tilde{H}+o(1)$ and has $\left|D_{x x}^{2} \tilde{u}\right| \leq \frac{C}{h^{2 / 3}}$. Since in each triangle the oscillation of the derivative is $O\left(h^{1 / 3}\right)$ we have the result, since

$$
H\left(D_{x} \phi_{u}, x\right) \leq \bar{H}+o(1)
$$

A corollary to the previous theorem is the following:

Corollary 1: Suppose $\xi_{h} \in \mathbb{R}^{n}$ is a supporting plane for $\bar{H}_{h}(P)$ that converges as $h \rightarrow 0$ to $\xi$. Then $\xi$ is a supporting hyperplane for $\bar{H}(P)$. As a consequence if $\bar{H}(P)$ is differentiable at $P$ then $\xi_{h}$ converges to the unique supporting hyperplane of $\bar{H}(P)$ at $P$.

\section{NUMERICAL IMPLEMENTATION}

We can make a further approximation, discretizing the spatial variable by computing the supremum only at the nodes $x_{i}$, which gives the minimax problem

$$
\min _{\phi \in C\left(T_{h}\right)} \max _{x_{i}} H\left(D_{x} \phi, x_{i}\right)
$$

for $x_{i}$ at the nodal points of the finite element space.

The minimax problem (5) is a finite dimensional nonlinear optimization problem which can by solved using standard optimization routines. We carried out the implementation in MATLAB, using the Optimization Toolbox.

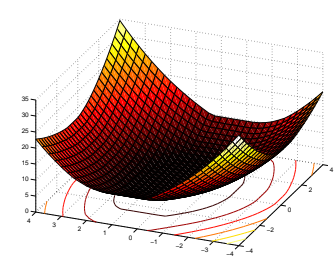

Fig. 2: $\bar{H}(P)$ for the double pendulum

\section{Computational Results}

\section{A. Strictly convex Hamiltonians}

We present two examples, the one-dimensional pendulum and the double pendulum.

Example 1 (one-dimensional pendulum): In this case the Hamiltonian is $H(p, x)=\frac{p^{2}}{2}-\cos 2 \pi x$. the result is presented in Figure 1, and agrees with the explicit formula for $\bar{H}$, which is known in this case.

Example 2 (Double pendulum): The double pendulum is a well known non-integrable system for which the effective Hamiltonian is not known. The Hamiltonian for the double pendulum is

$\frac{p_{x}^{2}-2 p_{x} p_{y} \cos (2 \pi(x-y))+2 p_{y}^{2}}{2-\cos ^{2}(2 \pi(x-y))}+2 \cos 2 \pi x+\cos 2 \pi y$.

The result is presented in Figure 2.

\section{B. Non strictly convex problems}

In this section we study several examples in which $H$ is convex, but not strictly convex, for which there is a viscosity solution of (HB).

Example 3 (Linear non-resonant): Consider the linear (nonresonant) Hamiltonian

$$
H(p, x)=\omega \cdot p+V(x, y) .
$$

Suppose $u$ is a smooth solution of (HB). Integrating the equation over $\mathbb{T}^{n}$ yields

$$
\bar{H}(0)=\int_{\mathbb{T}^{n}} V,
$$

and so $\bar{H}(P)=\bar{H}(0)+\omega \cdot P$.

For the example $u_{x}+\sqrt{2} u_{y}+\cos (2 \pi x)$ we obtained $D_{P} \bar{H}=(1, \sqrt{2})$ and $\bar{H}(0,0)=0$. In this (linear) case the optimization routine converged very quickly.

Example 4 (Vakonomic): Finally, we study an example of a non-strictly convex Hamiltonian which satisfies commutation relations related to vakonomic mechanics [AKN97],

$$
H(p, x)=\frac{\left|f_{1} \cdot D u\right|^{2}}{2}+\frac{\left|f_{2} \cdot D u\right|^{2}}{2}+V(x, y)
$$

in which the vector fields $f_{1}, f_{2}$ do not span $\mathbb{R}^{2}$ in every point but when we consider the commutator $\left[f_{1}, f_{2}\right]$ we have that 


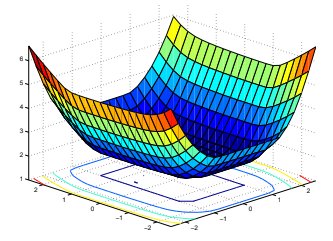

Fig. 3: $\bar{H}(P)$ for the Vakonomic Hamiltonian.

$f_{1}, f_{2},\left[f_{1}, f_{2}\right]$ span $\mathbb{R}^{2}$ in every point. In this situation (HB) has Hölder continuous viscosity solutions [EJ89], [Gom01a].

We chose $f_{1}=(0,1)$, and $f_{2}=(\cos 2 \pi y, \sin 2 \pi y)$, so that $f_{1}, f_{2},\left[f_{1}, f_{2}\right]$ always span $\mathbb{R}^{2}$. Therefore there is a Hölder continuous viscosity solution. The potential is $V(x, y)=$ $\cos 2 \pi x+\sin 2 \pi(x-y)$ The result is presented in Figure 3.

\section{Non-existence of viscosity solutions}

There are situations where there do not exist viscosity solutions to (HB), but $\bar{H}$ can still be defined by solving a more general problem, see [BS00], [BS01] and [LS03]. In some of these situations, the solution of the minimax problem (1) may exist and give a consistent result.

We work out two interesting examples and try to explain the results obtained numerically.

The problem

$$
\alpha u^{\alpha}+H\left(P+D_{x} u^{\alpha}, x\right)=0,
$$

which (when $\alpha \neq 0$ ) has a unique solution is considered in [LS03]. Sending $\alpha \rightarrow 0$ gives the effective Hamiltonian

$$
\bar{H}(P) \equiv \lim _{\alpha \rightarrow 0} \alpha u^{\alpha} \text {. }
$$

Proposition 5: Let $u^{\alpha}$ be a solution of (8), and suppose $\alpha u^{\alpha}$ converges uniformly to a constant number $\bar{H}(P)$. Then

$$
\bar{H}(P)=\lim _{\alpha \rightarrow 0} \alpha u^{\alpha}=\inf _{\phi} \sup _{x \in \mathbb{T}^{n}} H\left(P+D_{x} \phi, x\right) .
$$

Proof: 1. Define $\bar{H}_{\alpha} \equiv-\alpha \min _{x} u^{\alpha}$ and

$$
v^{\alpha} \equiv u^{\alpha}+\frac{\bar{H}_{\alpha}}{\alpha},
$$

so that $\min _{x} v^{\alpha}=0$. We will demonstrate $\bar{H}_{\alpha} \rightarrow \bar{H}$. We have

$$
\begin{aligned}
\bar{H} & =\lim _{\alpha \rightarrow 0} H\left(P+D_{x} u^{\alpha}, x\right)=\lim _{\alpha \rightarrow 0}-\alpha u^{\alpha} \\
& =\lim _{\alpha \rightarrow 0} \alpha\left(u^{\alpha}-\min _{x} u^{\alpha}\right)+\alpha \min _{x} u^{\alpha}=\bar{H}_{\alpha}
\end{aligned}
$$

2. Let $v_{\alpha}^{\epsilon}$ denote the $\sup$ convolution of $v_{\alpha}$ and let $\phi=\eta_{\epsilon} * v_{\alpha}^{\epsilon}$. Then $H\left(D_{x} \phi, x\right) \leq \bar{H}_{\alpha}+O(\epsilon)$. Therefore $\inf _{\phi} \sup _{x \in \mathbb{T}^{n}} H\left(D_{x} \phi, x\right) \leq \bar{H}_{\alpha} \rightarrow \bar{H}$.

3. Now let $e_{\alpha}=\sup _{x} \alpha v_{\alpha}$, which converges to 0 .

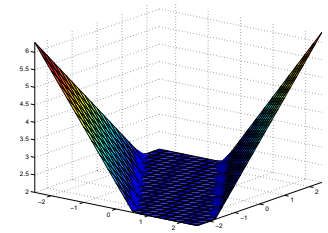

Fig. 4: $\bar{H}(P)$ for the quasi-periodic Hamiltonian.

Let $\phi$ be any function. Then $v_{\alpha}-\phi$ has a local minimum at a point $x_{0}$. At this point

$$
\alpha v_{\alpha}\left(x_{0}\right)+H\left(D_{x} \phi\left(x_{0}\right), x_{0}\right) \geq \bar{H}_{\alpha},
$$

and so $\sup _{x \in \mathbb{T}^{n}} H\left(D_{x} \phi, x\right) \geq \bar{H}_{\alpha}-e_{\alpha} \rightarrow \bar{H}$. Therefore $\inf _{\phi} \sup _{x \in \mathbb{T}^{n}} H\left(D_{x} \phi, x\right) \geq \bar{H}$.

Example 5 (Quasiperiodic Hamiltonians): We give an example from [LS03] where there is no viscosity solution to (HB), but where $\bar{H}(P)$ can be determined from (9). Let

$$
H\left(p_{x}, p_{y}, x, y\right)=\left|p_{x}+\alpha p_{y}\right|+\sin (x)+\sin (y)
$$

with $\alpha$ irrational.

We computed $\bar{H}(P)$ numerically from (1). The results are presented in Figure 4.

Example 6 (Linear resonant): Resonant linear Hamiltonians (6) may fail to have a viscosity solution. An example is

$$
(0,1) \cdot D u+\sin (2 \pi x)=\bar{H} .
$$

The formula (7) yields $\bar{H}(0)=0$ if there were a solution of (HB). However, we have

$$
\inf _{\phi} \sup _{x} H\left(D_{x} \phi, x\right)=1 .
$$

In fact, let $\phi$ be an arbitrary periodic function. Set $x_{0}=1 / 4$, so that $\sin 2 \pi x_{0}=1$. Then $\phi\left(x_{0}, y\right)$ is a periodic function of $y$ and so $D_{y} \phi\left(x_{0}, y\right)=0$ at some $y=y_{0}$. Thus

$$
\sup _{x} H\left(D_{x} \phi, x\right) \geq H\left(D_{x} \phi\left(x_{0}, y_{0}\right), x_{0}, y_{0}\right)=1 .
$$

Numerically we obtained $D_{P} \bar{H}=(0,1)$ and $\bar{H}(0,0)=1$.

\section{REFERENCES}

[AI01] O. Alvarez and H. Ishii. Hamilton-Jacobi equations with partial gradient and application to homogenization. Comm. Partial Differential Equations, 26(5-6):983-1002, 2001.

[AKN97] V. I. Arnold, V. V. Kozlov, and A. I. Neishtadt. Mathematical aspects of classical and celestial mechanics. Springer-Verlag, Berlin, 1997. Translated from the 1985 Russian original by A. Iacob, Reprint of the original English edition from the series Encyclopaedia of Mathematical Sciences [Dynamical systems. III, Encyclopaedia Math. Sci., 3, Springer, Berlin, 1993; MR 95d:58043a].

[Ari97] Mariko Arisawa. Ergodic problem for the Hamilton-JacobiBellman equation. I. Existence of the ergodic attractor. Ann. Inst. H. Poincaré Anal. Non Linéaire, 14(4):415-438, 1997. 
[Ari98] Mariko Arisawa. Ergodic problem for the Hamilton-JacobiBellman equation. II. Ann. Inst. H. Poincaré Anal. Non Linéaire, 15(1):1-24, 1998.

[BS00] G. Barles and Panagiotis E. Souganidis. On the large time behavior of solutions of Hamilton-Jacobi equations. SIAM $J$. Math. Anal., 31(4):925-939 (electronic), 2000.

[BS01] G. Barles and P. E. Souganidis. Space-time periodic solutions and long-time behavior of solutions to quasi-linear parabolic equations. SIAM J. Math. Anal., 32(6):1311-1323 (electronic), 2001.

[CDI01] I. Capuzzo-Dolcetta and H. Ishii. On the rate of convergence in homogenization of Hamilton-Jacobi equations. Indiana Univ. Math. J., 50(3):1113-1129, 2001.

[CIPP98] G. Contreras, R. Iturriaga, G. P. Paternain, and M. Paternain. Lagrangian graphs, minimizing measures and Mañé's critical values. Geom. Funct. Anal., 8(5):788-809, 1998.

[Con95] M. Concordel. Periodic homogenization of Hamilton-Jacobi equations. PhD thesis, UC Berkeley, 1995.

[E99] Weinan E. Aubry-Mather theory and periodic solutions of the forced Burgers equation. Comm. Pure Appl. Math., 52(7):811828, 1999.

[EG01a] L. C. Evans and D. Gomes. Effective Hamiltonians and averaging for Hamiltonian dynamics. I. Arch. Ration. Mech. Anal., 157(1):1-33, 2001.

[EG01b] L. C. Evans and D. Gomes. Effective Hamiltonians and averaging for Hamiltonian dynamics II. To appear in the Arch. Ration. Mech. Anal., 2001.

[EJ89] L. C. Evans and M. R. James. The Hamilton-Jacobi-Bellman equation for time-optimal control. SIAM J. Control Optim., 27(6):1477-1489, 1989.

[EMS95] Pedro F. Embid, Andrew J. Majda, and Panagiotis E. Souganidis. Comparison of turbulent flame speeds from complete averaging and the G-equation. Phys. Fluids, 7(8):2052-2060, 1995.

[Fat97a] Albert Fathi. Solutions KAM faibles conjuguées et barrières de Peierls. C. R. Acad. Sci. Paris Sér. I Math., 325(6):649-652, 1997.

[Fat97b] Albert Fathi. Théorème KAM faible et théorie de Mather sur les systèmes lagrangiens. C. R. Acad. Sci. Paris Sér. I Math., 324(9):1043-1046, 1997.

[Fat98a] Albert Fathi. Orbite hétéroclines et ensemble de Peierls. C. R. Acad. Sci. Paris Sér. I Math., 326:1213-1216, 1998.

[Fat98b] Albert Fathi. Sur la convergence du semi-groupe de Lax-Oleinik. C. R. Acad. Sci. Paris Sér. I Math., 327:267-270, 1998

[Gom01a] D. Gomes. Hamilton-Jacobi methods for Vakonomic Mechanics. Preprint, 2001.

[Gom01b] D. Gomes. Viscosity solutions of Hamilton-Jacobi equations, and asymptotics for Hamiltonian systems. J. Cal. of Var. (http://dx.doi.org/10.1007/s005260100106), 2001.

[KBM01] B. Khouider, A. Bourlioux, and A. J. Majda. Parametrizing the burning speed enhancement by small-scale periodic flows. I. Unsteady shears, flame residence time and bending. Combust. Theory Model., 5(3):295-318, 2001.

[LPV88] P. L. Lions, G. Papanicolao, and S. R. S. Varadhan. Homogeneization of Hamilton-Jacobi equations. Preliminary Version, 1988.

[LS03] Pierre-Louis Lions and Panagiotis E. Souganidis. Correctors for the homogenization of Hamilton-Jacobi equations in the stationary ergodic setting. Comm. Pure Appl. Math., 56(10):1501$1524,2003$.

[Mat89] John N. Mather. Minimal measures. Comment. Math. Helv, 64(3):375-394, 1989.

[Mat91] John N. Mather. Action minimizing invariant measures for positive definite Lagrangian systems. Math. Z., 207(2):169-207, 1991.

[Qia01] J. Qian. A note on a numerical method for effective Hamiltonians. Preprint, 2001 